This item was submitted to Loughborough's Research Repository by the author.

Items in Figshare are protected by copyright, with all rights reserved, unless otherwise indicated.

\title{
Airborne behaviour monitoring using Gaussian processes with map information
}

PLEASE CITE THE PUBLISHED VERSION

http://dx.doi.org/10.1049/iet-rsn.2012.0255

\section{PUBLISHER}

(C) Institution of Engineering and Technology (IET)

\section{VERSION}

AM (Accepted Manuscript)

\section{PUBLISHER STATEMENT}

This work is made available according to the conditions of the Creative Commons Attribution-NonCommercialNoDerivatives 4.0 International (CC BY-NC-ND 4.0) licence. Full details of this licence are available at: https://creativecommons.org/licenses/by-nc-nd/4.0/

\section{LICENCE}

CC BY-NC-ND 4.0

\section{REPOSITORY RECORD}

Oh, Hyondong, Hyo-Sang Shin, Seungkeun Kim, Antonios Tsourdos, and Barry A. White. 2015. "Airborne Behaviour Monitoring Using Gaussian Processes with Map Information". figshare. https://hdl.handle.net/2134/17840. 


\title{
Airborne Behaviour Monitoring Using \\ Gaussian Processes with Map Information
}

\author{
Hyondong Oh, Hyo-Sang Shin, Seungkeun Kim, \\ Antonios Tsourdos, and Brian A. White
}

\begin{abstract}
This paper proposes an airborne behaviour monitoring methodology of ground vehicles based on a statistical learning approach with domain knowledge given by road map information. To monitor and track the moving ground target using UAVs aboard a moving target indicator, an interactive multiple model (IMM) filter is firstly applied. The IMM filter consists of an on-road moving mode using a roadconstrained filter and an off-road moving mode using a conventional filter. Mode probability is also calculated from the IMM filter, and it provides deviation of the vehicle from the road. Then, a novel hybrid algorithm for anomalous behaviour recognition is developed using a Gaussian process regression on velocity profile along the one-dimensionalised position of the vehicle, as well as the deviation of the vehicle. To verify the feasibility and benefits of the proposed approach, a numerical simulation is performed using realistic car trajectory data in a city traffic.
\end{abstract}

\section{Index Terms}

Behaviour Monitoring, Gaussian processes, interactive multiple model filter, map information, target tracking, unmanned aerial vehicles.

\section{INTRODUCTION}

Since airborne monitoring using UAVs (unmanned aerial vehicles) can improve situational awareness and help human operators to lead appropriate decision making, it will enable many new

Hyondong Oh, Hyo-Sang Shin, Antonios Tsourdos, and Brian A. White are with Department of Engineering Physics, Cranfield University, Cranfield, UK (e-mail: h.oh; h.shin; a.tsourdos; b.a.white@cranfield.ac.uk)

Seungkeun Kim is with Department of Aerospace Engineering, Chungnam National University, 99 Daehak-ro, Yuseong-gu, Daejeon 305-764, Korea (email: skim78@cnu.ac.kr). 
and innovative UAV applications. Specific applications under consideration include, but are not limited: border patrol, search and rescue, surveillance, and law enforcement. In particular, UAV surveillance with an onboard GMTI (ground moving target indicator) radar or SAR (synthetic aperture radar) can provide a certain level of accurate estimation of massive sets of targets and consequently allow detection of possible threats [1], [2], [3]. However, the operators still need to analyse the gathered data and construct a picture of events to detect suspicious behaviours. This requires several highly-skilled human operators which is expensive and unsustainable under deluge of data and information. Hence, there is a strong need to develop high-level analysis algorithms to process target information and detect anomalous behaviours so that some of the human operator's workload resides with the vehicle.

Activity modelling and automatic anomaly detection tasks are non-trivial since complex activity patterns in a dynamic environment involve multiple objects interacting with each other over space and time, whilst anomalies are often rare, ambiguous and can be easily confused with sensor noise. In particular, anomalous behaviours of a ground vehicle are closely related to driving behaviours such as aggressive, drowsy, or intoxicated lane crossing manoeuvres [4], [5]. Also, location where a vehicle is moving, speed, and regional activity patterns (whose characteristics are often defined by the combination of location and speed) [6] are other important aspects to identify anomalous behaviours.

Detecting anomalous behaviours can be classified into two routes: First approach codifies the behaviours using experience and domain knowledge of experts and the behaviours are learned from data in second approach [7]. Roy [8] proposed a rule-based expert system implementing automated rule-based reasoning in support of maritime anomaly detection. Jasinevicius and Petrauskas [9] also used a rule-based expert map but combining with fuzzy logic for a port security system. Kim et al. [10] proposed the use of the trajectory classification codified with the string of numbers and string matching to suspicious behaviour references pre-defined from expert knowledge. For learning approach for anomaly detection, many works have been performed using parametric models such as Bayesian network [11], [12] and hidden Markov models [13]. In order to alleviate the complexity of the problem, Gaussian process (GP) models are also applied. The GPs can be considered as a Gaussian distribution over functions specified by its mean and covariance function. Predictive distributions are inferred from the regression models to compare with the actual observations providing anomaly measure. Will et al. [7] used the Kd-tree GPs to 
model shipping behaviour and maritime anomaly detection. Loy et al. [6] also applied the GPs for activity modelling and real-time anomaly detection of a ground traffic flow using surveillance video footage.

This study proposes an airborne behaviour monitoring methodology of ground vehicles using both of a learning approach based on GPs and domain knowledge provided from road map information. Compared to commonly used parametric models such as hidden Markov models, since GPs are not a parametric model, users do not need to be concerned about if it is possible for the model to fit the data [6]. The use of a flexible, non-parametric model alleviates the difficulty of selecting appropriate model complexity encountered in parametric models. GP models need fewer parameters; they are thus less likely to overfit given sparse data of moving vehicles. The velocity profile of the vehicle with respect to its position is one of principal factors which provide measures of the suspicious and abnormal behaviours. Therefore, the proposed Gaussian process regression is implemented on the velocity profile along the one-dimensional representation of the vehicle position. Since some vehicles could move on off-road terrain to avoid a police checkpoint or to closely monitor a particular place, temporal probability of on/off-road modes is another important source for abnormal behaviours detection. In order to obtain this mode probability whilst enabling monitoring of the moving ground target, an interactive multiple model (IMM) filter [14] is applied. The proposed IMM filter comprises an on-road moving mode using a roadconstrained filter and an off-road moving mode using a conventional filter so that both on and off mode probabilities (which are complementary to each other) can be obtained. In this study, in order to combine these two approaches, a novel hybrid algorithm is also developed based on the joint probability theory.

The key innovation of the proposed approach lies in the hybrid algorithm which integrates the two different philosophies and inherently brings several advantages. The proposed algorithm does not require to define a specific behaviour for suspicious behaviour recognition. Exploiting the velocity profile on a specific road from statistical traffic data allows consideration of the road conditions such as slope, roughness or curve. Moreover, anomaly measure on the vehicle behaviours can be obtained by evaluating the quality of predictions from the deviation of the actual target data from the predictive distribution. Since these anomaly scores are augmented with the on/off-road mode probability, the proposed algorithm can also complement the weakness of relying on the learning data which is difficult to incorporate a certain anomalous behaviour 
aspect.

The overall structure of this paper is given as: Section II explains a road-constrained IMM filter design including the definition of ground target and sensor model considered in this study. Section III introduces the 1-D representation of the position on the road of interest, and proposes a novel anomalous behaviour detection scheme combining predictive log-likelihood from Gaussian process regression and the off-road mode probability. Section IV presents numerical simulation results of behaviour monitoring scenario using realistic ground vehicle trajectory data. Lastly, conclusions and future works are given in Section V. The overall procedure of the behaviour monitoring of ground vehicles using UAVs is shown in Fig. 1.

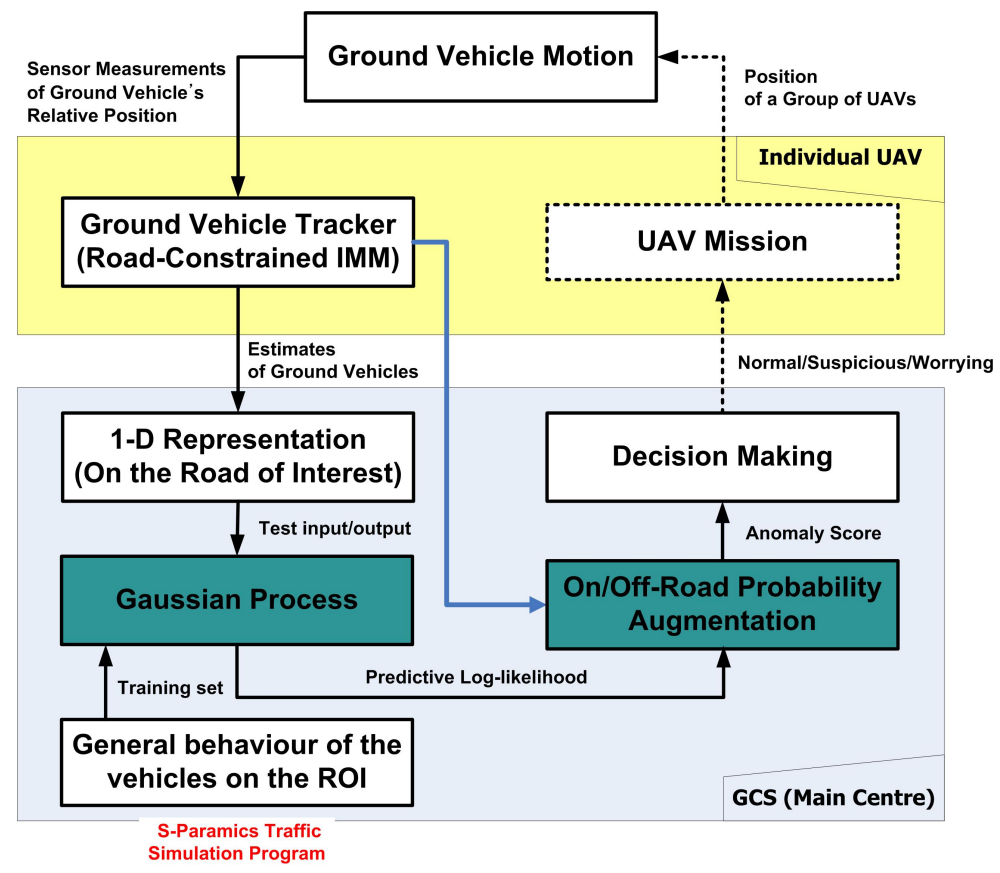

Fig. 1. Overall flow chart of behaviour monitoring

\section{ROAD-CONSTRAINED IMM FILTER}

This section presents models of the sensor and the ground target required for the target tracking. In addition, the road-constrained filter design is introduced using a road approximation technique and the IMM filter. 


\section{A. Ground target and sensor model}

This study considers acceleration dynamics to apply it to tracking of the moving ground vehicle. The model regards the target acceleration as a process correlated and exponentially decreasing in time, which means if there is a certain acceleration rate at a time $t$, then it is likely to be the same jerk also at a time instant $t+\tau$ as [15], [16]:

$$
\mathbf{x}_{k}^{t}=F_{k} \mathbf{x}_{k-1}^{t}+\eta_{k}
$$

where $\mathbf{x}_{k}^{t}=\left(x_{k}^{t}, \dot{x}_{k}^{t}, \ddot{x}_{k}^{t}, y_{k}^{t}, \dot{y}_{k}^{t}, \ddot{y}_{k}^{t}\right)^{T}, \eta_{k}$ is a process noise which represents the acceleration characteristics of the target, and the state transition matrix $F_{k}$ can be represented as:

$$
F_{k}=\left[\begin{array}{cccccc}
1 & T_{s} & \Phi & 0 & 0 & 0 \\
0 & 1 & \frac{\left(1-e^{-\alpha T_{s}}\right)}{\alpha} & 0 & 0 & 0 \\
0 & 0 & e^{-\alpha T_{s}} & 0 & 0 & 0 \\
0 & 0 & 0 & 1 & T_{s} & \Phi \\
0 & 0 & 0 & 0 & 1 & \frac{\left(1-e^{-\alpha T_{s}}\right)}{\alpha} \\
0 & 0 & 0 & 0 & 0 & e^{-\alpha T_{s}}
\end{array}\right]
$$

where $\Phi=\left(e^{-\alpha T_{s}}+\alpha T_{s}-1\right) / \alpha^{2}$, and $\alpha$ is a correlation parameter which allows for the modelling of the different classes of targets: small $\alpha$ for targets with relatively slow manoeuvres and high $\alpha$ for targets with fast and evasive ones. The details of the covariance matrix $Q_{k}$ of the process noise $\eta_{k}$ can be found in [15].

It is assumed that the UAV is equipped with a GMTI radar to localise the position of target. Since the measurement of GMTI sensor is composed of range and azimuth of the target with respect to the radar location, the actual measurements are the relative range, $r$, and azimuth, $\phi$, with respect to the position of the UAV airborne. The radar measurement $(r, \phi)^{T}$ can be defined as the following nonlinear relation using the target position $\left(x_{k}^{t}, y_{k}^{t}\right)^{T}$ and the UAV position $\left(x_{k}, y_{k}\right)^{T}$ as:

$$
\mathbf{z}_{k}=\left(\begin{array}{c}
r_{k} \\
\phi_{k}
\end{array}\right)=h\left(\mathbf{x}_{k}^{t}\right)+\nu_{k}=\left(\begin{array}{c}
\sqrt{\left(x_{k}^{t}-x_{k}\right)^{2}+\left(y_{k}^{t}-y_{k}\right)^{2}} \\
\tan ^{-1} \frac{y_{k}^{t}-y_{k}}{x_{k}^{t}-x_{k}}
\end{array}\right)+\nu_{k}
$$

where $\nu_{k}$ is a measurement noise vector, and its noise covariance matrix is defined as:

$$
V\left[\nu_{k}\right]=R_{k}=\left[\begin{array}{cc}
\sigma_{r}^{2} & 0 \\
0 & \sigma_{\phi}^{2}
\end{array}\right]
$$




\section{B. Road-constrained estimation}

To make use of road-map information for the estimation of a target travelling on a road, it is required to express the road-map as a certain type of mathematical equations approximating the real road from a given geographical database. To do so, this study uses constant curvature segments. In this approach, assuming that some of vertices on the road can be obtained, those vertices are connected by line segments of constant curvature with $\mathcal{C}^{2}$ contact at the vertices. The curved line between the vertices is to represent the curved nature of the real road. To ensure the $\mathcal{C}^{2}$ constraints at both positions, an intermediate vertex is introduced such that the line segment is replaced by two arc segments of different curvature. The mathematical details of the construction of the line curvature between vertices are described in [17]. The entire road-map can then be modelled by a set of road segments $r_{i}, i \in\left\{1, \ldots, n_{r}\right\}$, and for each road segment, the centre position of the road curve and its curvature are given by the approximation algorithm.

Figure 2 shows sample road-network of Devizes, Wiltshire, United Kingdom with GIS satellite data. Given information for road of interest as blue line in this case, Fig. 3 shows the approximated road segments. As shown in Fig. 3(b), the more vertices are used, the better approximated road is obtained. However, since too many road segments might cause performance degradation in the constrained estimation, the appropriate number of vertices on the road needs to be determined considering the road-network structure.

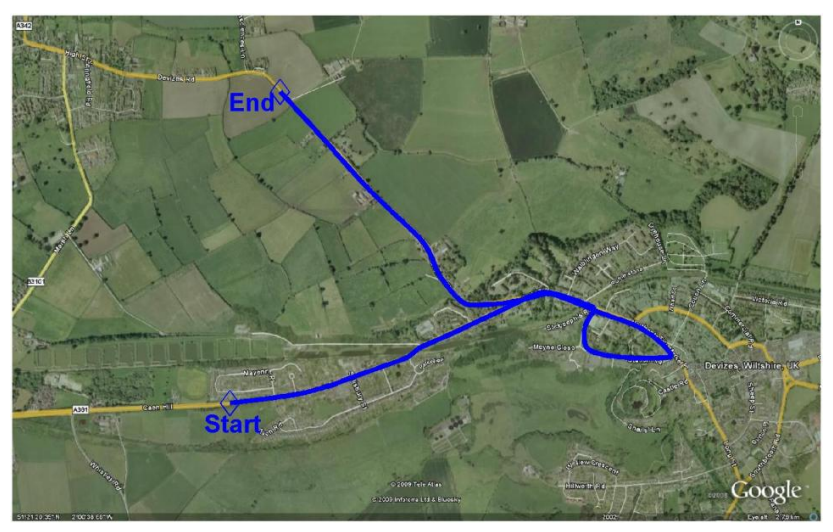

Fig. 2. Sample road network with GIS satellite data overlaid (Google Map)

Now, assuming that the ground vehicle moves along given road-map consisting of $n$ road segments, the 2-D position of the vehicle should lie on the one of roads. This can be expressed 


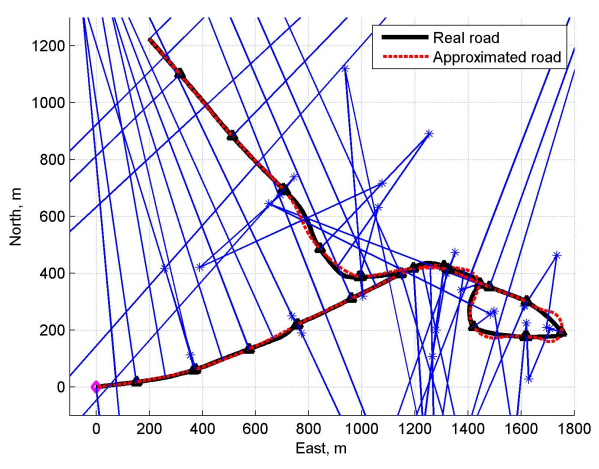

(a) Loosely represented roads

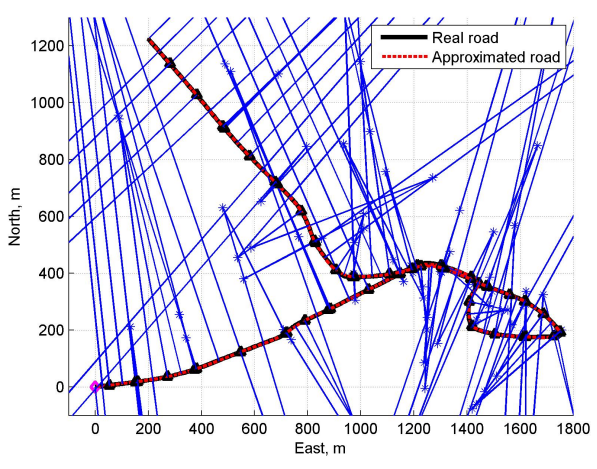

(b) Densely represented roads

Fig. 3. Road approximation using constant curvature segments

as the following constraint:

$$
r_{i}\left(x_{k}^{t}, y_{k}^{t}\right)=0
$$

where $r_{i}(\cdot)$ denotes the $i$-th road segment which can be modeled as straight line, arc, or polynomials. In this study since the road is approximated using constant curvature segments as explained earlier, the road constraint can be obtained as:

$$
r_{i}\left(x_{k}^{t}, y_{k}^{t}\right)=\left(x_{k}^{t}-x_{i, c t}\right)^{2}+\left(y_{k}^{t}-y_{i, c t}\right)^{2}-\left(\frac{1}{\kappa_{i}}\right)^{2}=0
$$

where $\left(x_{i, c t}, y_{i, c t}\right)$ and $\kappa_{i}$ are the centre position and the curvature of the $i^{t h}$ road segment, respectively. Typically, there are two ways to deal with the road constraint in Kalman filtering framework. One is to use the road as equality constraints [18], and the other is to use the concept of directional process noise [19], which represents uncertainty components along and orthogonal to the road. This study uses a pseudo-measurement method, one of the constrained Kalman filtering algorithms, which treats the equality constraints as additional fictitious or pseudo-measurement [20]. Unlike the other approaches such as maximum probability method and projection method [21], this has the advantage which allows us to consider the degree of constraint adherence by the magnitude of the additional pseudo-measurement noise variance. Pseudo-measurement model using road constraints can be written as:

$$
z_{k}^{r_{i}}=h_{r_{i}}\left(\mathbf{x}_{k}^{t}\right)+\nu_{k}^{r_{i}}
$$


where $z_{k}^{r_{i}}=0, h_{r_{i}}\left(\mathbf{x}_{k}^{t}\right)=r_{i}\left(\mathbf{x}_{k}^{t}\right)$, and $\nu_{k}^{r_{i}}$ is assumed to be zero mean white Gaussian noise with its covariance $R_{k}^{r_{i}}=\left(\sigma_{r}^{\text {road }}\right)^{2}$ which accounts for the uncertainty of road constraints. Then, the previous real measurement model Eq. (3) is augmented by adding the pseudo-measurement as:

$$
\mathbf{z}_{k}^{a}=h_{a}\left(\mathbf{x}_{k}^{t}\right)+\nu_{k}^{a}
$$

where $\mathbf{z}_{k}^{a}=\left[\begin{array}{ll}\mathbf{z}_{k} & z_{k}^{r_{i}}\end{array}\right]^{T}, h_{a}\left(\mathbf{x}_{k}^{t}\right)=\left[h\left(\mathbf{x}_{k}^{t}\right) h_{r_{i}}\left(\mathbf{x}_{k}^{t}\right)\right]^{T}$, and $\nu_{k}^{a}=\left[\begin{array}{ll}\nu_{k} & \nu_{k}^{r_{i}}\end{array}\right]^{T}$. The measurement noise covariance is also augmented as $R_{k}^{a}=\operatorname{diag}\left(R_{k}, R_{k}^{r_{i}}\right)$. Considering $h_{a}\left(\mathbf{x}_{k}^{t}\right)$ in the measurement equation is nonlinear, the localisation of a target can be designed by using the extended Kalman filter $(\mathrm{EKF})[22]$ as:

Time update

$$
\begin{aligned}
& \mathbf{x}_{k \mid k-1}^{t}=F_{k} \mathbf{x}_{k-1 \mid k-1}^{t} \\
& P_{k \mid k-1}=F_{k} P_{k-1 \mid k-1} F_{k}^{T}+Q_{k}
\end{aligned}
$$

Measurement update

$$
\begin{aligned}
v_{k} & =\mathbf{z}_{k}^{a}-h_{a}\left(\mathbf{x}_{k \mid k-1}^{t}\right) \\
S_{k} & =H_{k} P_{k \mid k-1} H_{k}^{T}+R_{k}^{a} \\
\mathbf{x}_{k \mid k}^{t} & =\mathbf{x}_{k \mid k-1}^{t}+P_{k \mid k-1} H_{k}^{T} S_{k}^{-1} v_{k} \\
P_{k \mid k} & =\left(I-P_{k \mid k-1} H_{k}^{T} S_{k}^{-1} H_{k}\right) P_{k \mid k-1} .
\end{aligned}
$$

The output matrix $H_{k}$ is a Jacobian of $h_{a}$ with respect to the time-update state $\mathbf{x}_{k \mid k-1}^{t}$. As a target is moving from one road segment to the other, an appropriate road on which the target is travelling is selected based on estimated or a priori target position, its error covariance and the road network information as:

$$
\left[\begin{array}{c}
x_{e}^{r_{i}}-x_{k \mid k-1}^{t} \\
y_{e}^{r_{i}}-y_{k \mid k-1}^{t}
\end{array}\right]^{T}\left[P_{k \mid k-1}^{p o s}\right]^{-1}\left[\begin{array}{c}
x_{e}^{r_{i}}-x_{k \mid k-1}^{t} \\
y_{e}^{r_{i}}-y_{k \mid k-1}^{t}
\end{array}\right]<\varepsilon
$$

where $\left(x_{e}^{r_{i}}, y_{e}^{r_{i}}\right)$ is the end position of $i$-th road segment, $\varepsilon$ is the gate threshold parameter, and $P_{k \mid k-1}^{p o s}$ is the position submatrix of the prediction covariance $P_{k \mid k-1}$. In this study, a current road segment is changed to the next one once above condition is satisfied for the simplicity. 


\section{IMM filter}

Although a normal vehicle tends to move along the road only, some of vehicles could move on off-road terrain for some reasons, e.g. to avoid a police checkpoint or to closely monitor a particular place. Therefore, an estimation algorithm should be able to recognise both off-road and on-road movement depending on the situation, rather than constraining the estimates onto the road at all times. To address this, the IMM filter [14] is applied combining an off-road mode using the conventional filter and on-road mode using a road-constrained filter as explained above.

Let $M_{k}=\left\{0,1, \cdots, N_{k}-1\right\}$ be the mode set of the IMM estimator in the interval $(k-1, k]$ and $\mu_{j, k}$ be the probability that mode is in effect during the above time interval as:

$$
\mu_{j, k}=p\left\{m_{k}=j \in M_{k} \mid \mathbf{Z}_{k}\right\}
$$

where $p\{\cdot\}$ denotes the probability, and $\mathbf{Z}_{k}$ is the measurement sequence through time $k$. Physically, $m_{k}$ represents the type of motion (off-road $m_{k}=0$ or on-road $m_{k}=m \in\left\{1,2, \cdots, N_{k}-\right.$ $1\}$ ), and this is modelled as a Markov process with a first-order homogeneous Markovian transition of modes:

$$
\pi_{i j, k}=p\left\{m_{k}=j \mid m_{k-1}=i\right\} \quad \forall i, j \in M_{k}
$$

where $\pi_{i j}$ is the Markov transition probability from mode $i$ to mode $j$. The IMM filter uses a recursive algorithm composed of the following four processes: interaction/mixing, filtering, model probability update, and estimate combination. The input to a filter matching to a certain mode is obtained by mixing the state estimates under the assumption that this particular mode is in effect at the present step. Then, a conventional filtering is performed in parallel. The model probabilities are updated based on model-conditional likelihood functions. Finally, overall states are estimated from the probabilistically weighted sum of outputs from each filter. The estimated states from the combination process along with the model probability will be used not only for tracking of ground vehicles but also behaviour recognition in the following section.

\section{BEHAVIOUR MONITORING}

This section firstly introduces a one-dimensional (1-D) representation of the position of the vehicle on the road to simplify the vehicle position on a specific road. Then, a novel anomalous behaviour detection scheme is proposed using a predictive log-likelihood from a Gaussian process regression, as well as the off-road mode probability. 


\section{A. 1-D representation of the position}

To represent the behaviour of the ground vehicle travelling on the road, a 1-D representation of the vehicle's position is introduced using the similar approach as in [23]. The ground target trajectory when in traverse of a road-network can be modelled as curved segments connected with arcs of different curvature, and a moving target can be located uniquely using the time, street name (a road segment), and street number (distance relative to a starting point on the street). This study assumes a width-less road since the road width is negligible considering resolution and error of the sensor onboard the UAV monitoring the ground vehicle from the air. Let us

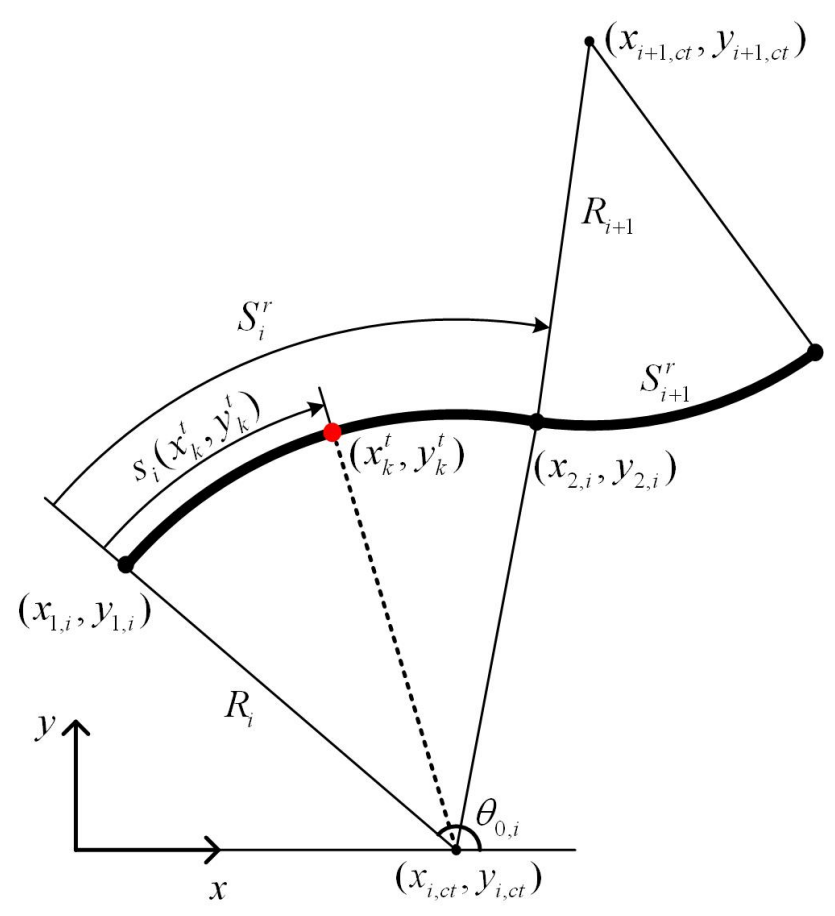

Fig. 4. 1-D representation of road segments

consider a circular arc characterised by starting point $\left(x_{1, i}, y_{1, i}\right)$, the ending point $\left(x_{2, i}, y_{2, i}\right)$, the centre of the arc $\left(x_{i, c t}, y_{i, c t}\right)$, and arc curvature $\kappa_{i}$ for $i^{t h}$ road (which can be obtained from the road approximation algorithm) as shown in Fig. 4. The location of the vehicle is determined by its distance from a starting point which will be referred as a mileage count [23]. Assuming multiple $n_{r}$ roads are connected in serial in the region of interest, a 2-D point $(x, y)$ on the 
curved road can be expressed using the mileage count $s_{i}$ as:

$$
\begin{aligned}
& x\left(s_{i}\right)=x_{i, c t}+R_{i} \cos \left(\theta_{0, i}+\frac{s_{i}}{R_{i}}\right) \\
& \left.y\left(s_{i}\right)=y_{i, c t}+R_{i} \sin \left(\theta_{0, i}+\frac{s_{i}}{R_{i}}\right)\right)
\end{aligned}
$$

where $0 \leq s_{i} \leq S_{i}^{r}, R_{i}=1 / \kappa_{i}$, and $\theta_{0, i}$ and $S_{i}^{r}$ are given by:

$$
\begin{gathered}
\theta_{0, i}=\tan ^{-1}\left(\frac{y_{1, i}-y_{i, c t}}{x_{1, i}-x_{i, c t}}\right) \\
S_{i}^{r}=R_{i} \cos ^{-1}\left[1-\frac{\left(x_{2, i}-x_{1, i}\right)^{2}+\left(y_{2, i}-y_{1, i}\right)^{2}}{2 R_{i}^{2}}\right] .
\end{gathered}
$$

The mileage $s_{i}$ of the ground vehicle at the position $\left(x_{k}^{t}, y_{k}^{t}\right)$ can be obtained using Eq. (18) inversely:

$$
\begin{aligned}
s_{i}\left(x_{k}^{t}, y_{k}^{t}\right) & =R_{i}\left[\cos ^{-1}\left(\frac{x_{k}^{t}-x_{i, c t}}{R_{i}}\right)-\theta_{0, i}\right] \\
& =R_{i}\left[\sin ^{-1}\left(\frac{y_{k}^{t}-y_{i, c t}}{R_{i}}\right)-\theta_{0, i}\right] .
\end{aligned}
$$

A normalised mileage count $\bar{s}$ of the vehicle position $\left(x_{k}^{t}, y_{k}^{t}\right)$ on $i^{\text {th }}$ road among $n_{r}$ roads of interest can be obtained as:

$$
\bar{s}\left(x_{k}^{t}, y_{k}^{t}\right)=\frac{\left(\sum_{j=1}^{i-1} S_{j}^{r}\right)+s_{i}\left(x_{k}^{t}, y_{k}^{t}\right)}{\sum_{j=1}^{n_{r}} S_{j}^{r}} .
$$

Note that the road on which the vehicle is can be determined by the road-constrained IMM filter. Even in case that the position of the vehicle is not exactly on the road, it can be constrained onto the road using the road-constrained filter as explained in the previous section. Using this 1-D representation, the behaviour of the vehicle on the road can simply be expressed as velocity, acceleration or any other quantities at specific position $\bar{s} \in[0,1]$ on the roads of interest.

\section{B. Gaussian process behaviour modelling}

This study uses a Gaussian process (GP) to model the behaviour of the vehicle. The GP can be viewed as a stochastic process or a Gaussian distribution over functions. The GPs are used to infer or predict function values at a finite set of test points using the observed data. Since GP is not a parametric model, users do not need to be concerned about whether it is possible for the model to fit the data or not. The GP for a regression problem [24] is briefly explained in the following. 
First of all, a standard regression model is defined as $y_{G P}=f(\mathbf{x})+\epsilon$, where $\mathbf{x}$ is an input vector (covariates) of dimension $D$, and $y_{G P}$ is a scalar output (dependent variable). The noise $\epsilon$ is assumed as an independent and identically distributed Gaussian distribution with zero mean and variance $\sigma^{2}$. Then, Gaussian process $f(\mathbf{x})$ is specified by its mean function $m(\mathbf{x})=\mathbb{E}[f(\mathbf{x})]$ and the covariance function $k\left(\mathbf{x}, \mathbf{x}^{\prime}\right)=\mathbb{E}\left[(f(\mathbf{x})-m(\mathbf{x}))\left(f\left(\mathbf{x}^{\prime}\right)-m\left(\mathbf{x}^{\prime}\right)\right)\right]$. Since this study assumes zero-mean GP, the process can be expressed as $f(\mathbf{x}) \sim G P\left(0, k\left(\mathbf{x}, \mathbf{x}^{\prime}\right)\right)$. A training set with $N_{o}$ observation is expressed as $\mathcal{D}=\left\{\left(\mathbf{x}_{n}, y_{G P, n}\right) \mid n=1, \cdots, N_{o}\right\}=\left\{\mathbf{X}, \mathbf{y}_{G P}\right\}$, and the following squared exponential covariance function is used:

$$
k\left(\mathbf{x}, \mathbf{x}^{\prime}\right)=\sigma_{f}^{2} \exp \left(-\frac{1}{2}\left(\mathbf{x}-\mathbf{x}^{\prime}\right)^{\top} \sum\left(\mathbf{x}-\mathbf{x}^{\prime}\right)\right)
$$

where $\sigma_{f}$ determines the magnitude, and $\sum=l^{-2} I$ is an isotropic covariance function.

Given a GP model with the covariance function above, the fitness of this model to the training set can be evaluated using the marginal likelihood conditioned on the hyper-parameters $\theta$ (which define the covariance function and the Gaussian noise) as:

$$
\log p\left(\mathbf{y}_{G P} \mid \mathbf{X}, \theta\right)=-\frac{1}{2} \mathbf{y}_{G P}^{\top} \mathbf{K}^{-1} \mathbf{y}_{G P}-\frac{1}{2} \log |\mathbf{K}|-\frac{N_{o}}{2}
$$

where $\mathbf{K}$ denotes the matrix of the covariances of all pairs of training points with $\mathbf{K}_{i j}=k\left(x_{i}, x_{j}\right)$. The hyper-parameters are optimised to provide good predictions using the partial derivatives of Eq. (25) with respect to the hyper-parameter using a conjugate gradient optimiser.

Given a training set $\mathcal{D}$ and a test input vector $\mathrm{x}_{*}$, the predictive distribution for Gaussian process regression is computed conditioning the joint Gaussian prior distribution of the observed output values $\mathbf{y}_{G P}$ and function values $f_{*}$ at the test locations on $\mathbf{X}$ and $\mathbf{x}_{*}$ as:

$$
f_{*} \mid \mathbf{X}, \mathbf{y}_{G P}, \mathbf{x}_{*} \sim \mathcal{N}\left(\bar{f}_{*}, \mathbb{V}\left[f_{*}\right]\right)
$$

where the mean and variance are defined as:

$$
\begin{aligned}
\bar{f}_{*} & =\mathbf{k}_{*}^{\top}\left(\mathbf{K}+\sigma_{n}^{2} I\right)^{-1} \mathbf{y}_{G P} \\
\mathbb{V}\left[f_{*}\right] & =k\left(\mathbf{x}_{*}, \mathbf{x}_{*}\right)-\mathbf{k}_{*}^{\top}\left(\mathbf{K}+\sigma_{n}^{2} I\right)^{-1} \mathbf{k}_{*}
\end{aligned}
$$

where $\mathbf{k}_{*}$ denotes the vector of covariance between the test and the training points.

In this study, the output $y_{G P}$ of GP model is a speed of the vehicle $\left(v^{t}=\sqrt{\left(\dot{x}^{t}\right)^{2}+\left(\dot{y}^{t}\right)^{2}}\right)$ observed at position on the roads of interest $\bar{s}\left(x^{t}, y^{t}\right) \in[0,1]$. It is assumed that the training data set is available using S-Paramics traffic simulation software [25] in advance, and test input 
and output come from the road-constrained IMM filter with sensor measurements of the UAV monitoring several ground vehicles in real time. Note that, although 1-D position $\bar{s}$ is used as an input variable currently, it can be extended to a vector including other relevant parameters such as time zone (day/night or weekday/weekend) or environmental factors (congestion or accidents) to more closely capture real world characteristics. One of limitations for using this approach is that training data should be given for the road (or region) of interest. However, if some of the road is important and under surveillance, traffic data could be readily available in advance or can be collected in real-time.

\section{Hybrid anomalous behaviour detection}

Given the prediction method, one can evaluate the quality of predictions measuring the deviation of the actual test data from the predictive distribution. This quality can be directly related to the anomalous behaviour detection since it provides how normally or abnormally the test vehicle behaves compared to the general vehicles. The simplest and intuitive way of measuring the quality is to compute the squared residual $S R_{k}=\left(v_{k}^{t}-\bar{f}\left(\bar{s}\left(x_{k}^{t}, y_{k}^{t}\right)\right)\right)^{2}$ between the mean prediction and the test output at each test point. To consider the predictive uncertainty additionally, a more conservative anomaly score in the form of negative log probability of the test output $v_{k}^{t}$ can be used as [24]:

$$
-\log p\left(v_{k}^{t} \mid \mathcal{D}, \bar{s}\right)=\frac{1}{2} \log \left(2 \pi \sigma_{*}^{2}\right)+\frac{\left(v_{k}^{t}-\bar{f}(\bar{s})\right)^{2}}{2 \sigma_{*}^{2}}
$$

where the predictive variance for GP regression is computed as $\sigma_{*}^{2}=\mathbb{V}\left[f_{*}\right]+\sigma_{n}^{2}$. The noise variance $\sigma_{n}^{2}$ is included since we are predicting the noisy output. Note that the more accurate the prediction of GP model is and the larger the predictive variance is, the less score Eq. (29) shows. This occurs when either prediction is correct (i.e. $v_{k}^{t}-\bar{f}(\bar{s}) \simeq 0$, or equivalently, test data fits with the normal behaviour from training data) or the model is uncertain about prediction with limited training data.

The log predictive probability in Eq. (29) can provide only a partial knowledge of the behaviour based on the velocity profile. In order to make a reliable a final decision on abnormal behaviour, other critical sources to recognise the abnormal behaviour need to be considered. Since the mode probability $\mu_{j, k}$ of the vehicle moving off-road terrain $(j=0)$ or on the roads of interest $(j=$ $1,2, \cdots, N_{k}-1$ ) is one of most important parameters for the abnormal behaviour recognition, 
this study proposes a novel hybrid behaviour recognition algorithm using not only Gaussian process but also the mode probability.

Assuming that probability from GP regression and mode probability are independent to each other (that is, the occurrence of one does not change the probability of the other occurring), an augmented abnormal score can be proposed using the negative log of the joint probability which is the product of the probabilities of each occurring as:

$$
\begin{aligned}
\text { score }_{k} & =-\log \left[p\left(v_{k}^{t} \mid \mathcal{D}, \bar{s}\right) \times\left(\sum_{j=1}^{N_{k}-1} \mu_{j, k}\right)^{\gamma}\right] \\
& =-\log \left[p\left(v_{k}^{t} \mid \mathcal{D}, \bar{s}\right) \times\left(1-\mu_{0, k}\right)^{\gamma}\right] \\
& =-\log p\left(v_{k}^{t} \mid \mathcal{D}, \bar{s}\right)-\gamma \log \left(1-\mu_{0, k}\right)
\end{aligned}
$$

where $\gamma$ is a weighting factor controlling the relative importance between GP predictive and on-road mode probability. From Eq. (30), it is clear that large values of the score represent that abnormal behaviour is likely to be occurring. If one of aforementioned probabilities (GP regression and on-road mode) is small, an augment abnormal score has a large value according to Eq. (30). As the both probabilities become small, since the product of them makes the joint probability smaller, an augment abnormal score gets bigger implying that the vehicle behaviour is likely suspicious or abnormal.

Note that an abnormal velocity does not necessarily incur off-road moving, whereas the deviation of the vehicle from the road can lead to an abnormal velocity caused by change of surface condition or traffic flow. Therefore, there is a need to investigate the conditional probability or dependence of those two events, rather than an independence assumption used in Eq. (30). Besides, to detect abnormal behaviour, a certain detection threshold needs to be determined considering the success and false alarm rate according the sensor characteristics and environments. These aspects remain for future works.

\section{NumERICAL Simulations}

This section carries out a numerical simulation using the proposed anomalous behaviour detection algorithm for a moving ground target using the UAV loitering the region of interest. The ground target trajectory is obtained from S-Paramics [25] traffic model of Devizes map at $2 \mathrm{~Hz}$. It is used to generate the GMTI measurements composed of relative range and azimuth 
angle having the white noise of $\left(\sigma_{r}, \sigma_{\phi}\right)=(7 \mathrm{~m}, 2 \mathrm{deg})$. Training data for GP regression is also obtained from the S-paramics model for 15 vehicles passing through the region of interest as shown in Fig. 5(a). Besides, it is assumed that there is only one road (but composed of multiple segments) around region of interest. In other words, we used a fixed mode set $M_{k}=\{0,1\}$ consisting of two measurement models with the common acceleration dynamics of the ground vehicle for the IMM filter: one is a conventional model for a vehicle moving on the off-road terrain as described in Eq. (3), and the other is a pseudo-measurement augmented model moving on the road of interest as in Eq. (8). The initial mode probability is set to $\left(\mu_{0}, \mu_{1}\right)=(0.2,0.8)$, and the Markov transition probability is given as:

$$
\left[\begin{array}{ll}
\pi_{11} & \pi_{12} \\
\pi_{21} & \pi_{22}
\end{array}\right]=\left[\begin{array}{ll}
0.99 & 0.01 \\
0.01 & 0.99
\end{array}\right]
$$

Note that the number of models can be changed to consider multiple roads at the intersection using a variable-structure IMM concept [26], [19].

Figure 5(b) shows the real roads of interest, their approximation, and the target trajectory from S-Paramics with a slight change of the route (on-road $\rightarrow$ de-tour to avoid police check point or monitor a certain facility more closely $\rightarrow$ on-road). Figures 6(a) 6(b) show Gaussian process regression result with training set and test data from the road-constrained IMM filter of the vehicle having normal speed, and the on/off-road mode probability. Depending on the similarity of velocity profile between the test ground vehicle and training mean value from GP, predictive log-likelihood varies accordingly as a blue line shown in Fig. 6(c). Besides, since the off-road mode probability is high when it is detouring the road, modified anomaly score from Eq. (30) has a high value. Figure 7 shows the simulations results for the same situation as the previous one but with the vehicle having abnormal speed during detour. In this case, since the speed of the test vehicle is quite different from the training data as well as off-road mode probability is high around $\bar{s}=0.6$, the anomaly score shows much larger value than the previous case representing that the anomalous or suspicious behaviour is highly likely to occur at that position.

\section{CONClusions And Future Work}

This paper proposed an airborne behaviour monitoring methodology of ground vehicles using a statistical approach based on Gaussian processes along with road map information. An interactive multiple model (IMM) filter is firstly applied combining an off-road mode using the conventional 


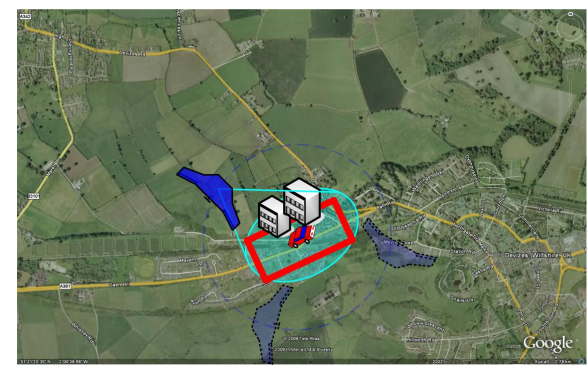

(a) Google map with region of interest

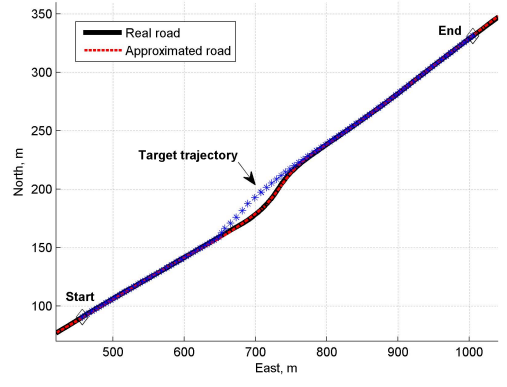

(b) Road approximation and target trajectory

Fig. 5. Road approximation and target trajectory in the region of interest

extended Kalman filter and on-road mode using a road-constrained filter. Then, a novel hybrid anomalous behaviour recognition algorithm was proposed using a Gaussian process regression on velocity profile with a one-dimensional representation of the position of the vehicle, as well as deviation of the vehicle location. A numerical simulation on monitoring of a suspicious ground target with a realistic map and trajectory data showed the feasibility of the proposed approach. Extension of the road-constrained IMM filter to the variable structure IMM filter concept will be followed as future work allowing the consideration of multiple roads at the intersection and different vehicle models. In addition, qualitative analysis will be performed generating a receiving operating characteristic (ROC) curve with a different detection threshold and parameters such as covariance function for GP model.

\section{REFERENCES}

[1] M. Ulmke and W. Koch, "Road-map assisted ground moving target tracking," IEEE Transactions on Aerospace and Electronic Systems, vol. 42, pp. 1264-1274, 2006.

[2] W. Koch and R. Klemm, "Ground target tracking with STAP radar," IEE Proceedings-Radar, Sonar and Navigation, vol. 148, pp. 173-185, 2001.

[3] D. Pastina and C. Spina, "Multi-feature based automatic recognition of ship targets in ISAR," IET Radar, Sonar and Navigation, vol. 3, pp. 406-423, 2009.

[4] C. F. Lin and A. G. Ulsoy, "Calculation of the time to lane crossing and analysis of its frequency distribution," in American Control Conference, 1995.

[5] C.-T. Lin, R.-C. Wu, S.-F. Liang, W.-H. Chao, Y.-J. Chen, and T.-P. Jung, "Eeg-based drowsiness estimation for safety driving using independent component analysis," IEEE Transactions on Circuits and Systems-I: Regular Papers, vol. 52, no. 12, pp. 2726-2738, 2005. 


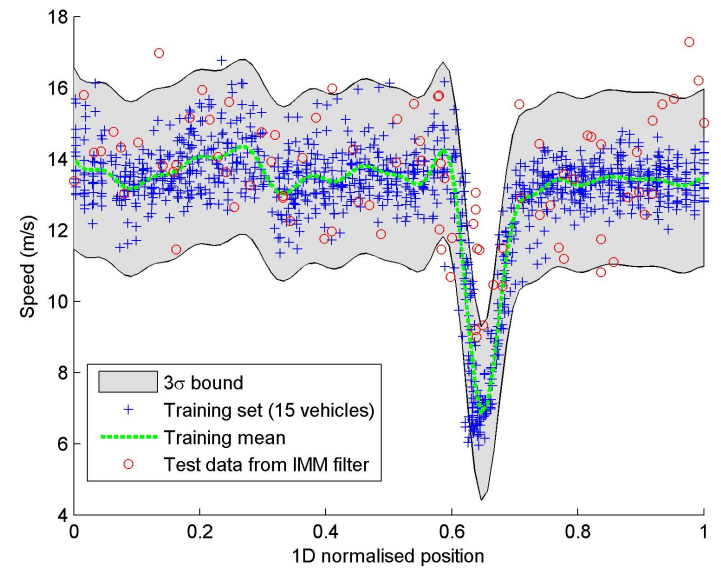

(a) Gaussian process regression

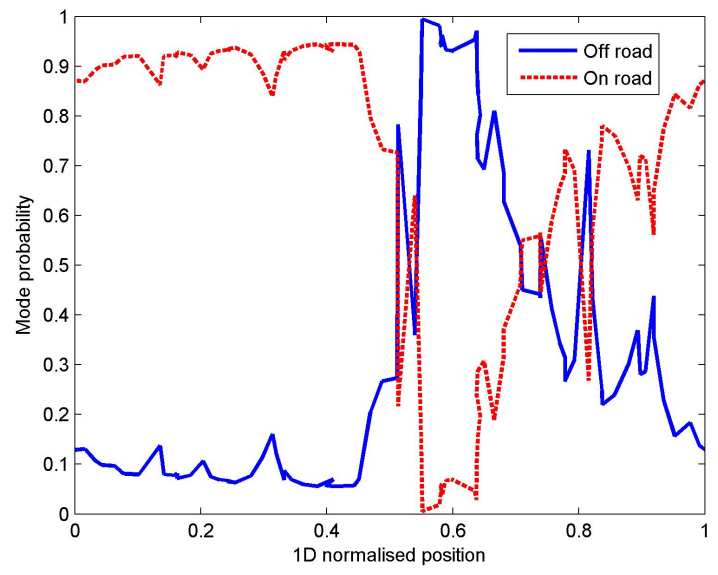

(b) On/off-road mode probability from IMM filter

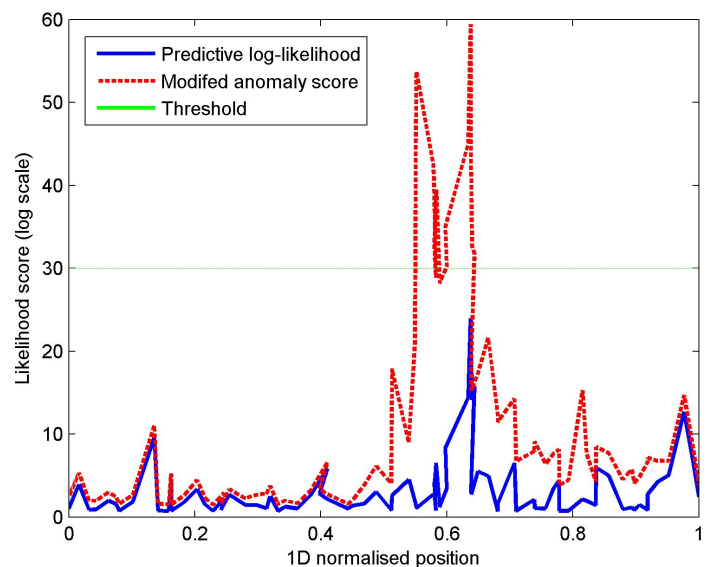

(c) Predictive log-likelihood and modified anomaly score $(\gamma=10)$

Fig. 6. Behaviour monitoring result with training set (15 vehicles from S-Paramics traffic simulator) and test data from road-constrained IMM filter with normal speed 


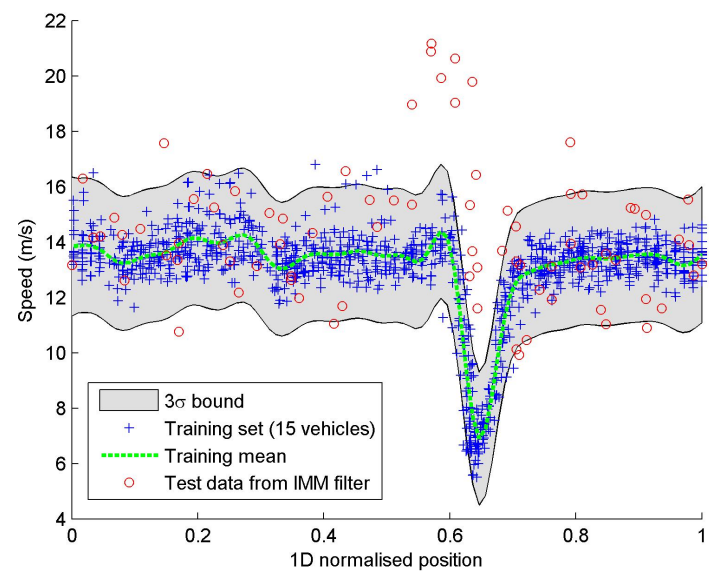

(a) Gaussian process regression

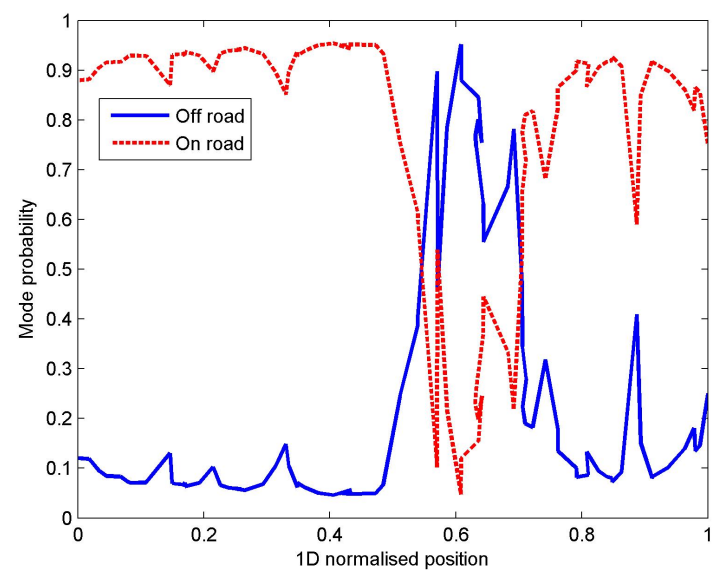

(b) On/off-road mode probability from IMM filter

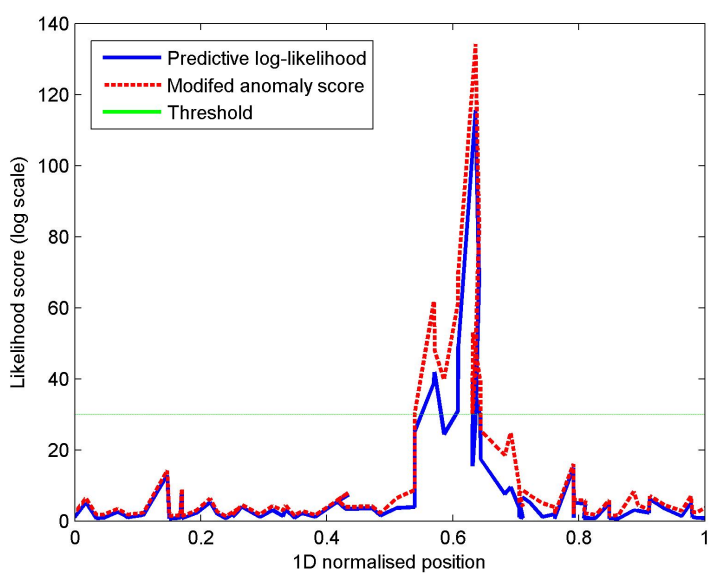

(c) Predictive log-likelihood and modified anomaly score $(\gamma=10)$

Fig. 7. Behaviour monitoring result with training set (15 vehicles from S-Paramics traffic simulator) and test data from road-constrained IMM filter with abnormal speed 
[6] C. Loy, T. Xiang, and S. Gong, "Modelling multi-object activity by gaussian processes," in British Machine Vision Conference, 2009.

[7] J. Will, L. Peel, and C. Claxton, "Fast maritime anomaly detection using kd-tree gaussian processes," in 2nd IMA Mathematics in Defence, (Defence Academy, Shrivenham, UK), 2011.

[8] J. Roy, "Rule-based expert system for maritime anomaly detection," in Sensors, and Command, Control, Communications, and Intelligence (C3I) Technologies for Homeland Security and Homeland Defense IX, Proc. SPIE 7666, 76662N, 2010.

[9] R. Jasinevicius and V. Petrauskas, "Fuzzy expert maps for risk management systems," in US/EU-Baltic International Symposium, 2008 IEEE/OES, 2008.

[10] S. Kim, R. Zbikowski, A. Tsourdos, and B. White, "Airborne monitoring of ground traffic behaviour for hidden threat assessment," in 13th International Conference on Information Fusion, (Endinburgh, UK), July 2010.

[11] F. Johansson and G. Falkman, "Detection of vessel anomalies - a bayesian network approach," in 3rd International Conference on Intelligent Sensors, Sensor Networks and Information, 2007.

[12] Y. Du, F. Chen, W. Xu, and Y. Li, "Recognizing interaction activities using dynamic bayesian networks," in ICPR, 2006.

[13] T. Duong, H. Bui, D. Phung, and S. Venkatesh, "Activity recognition and abnormality detection with the switching hidden semi-markov model," in International Conference on Computer Vision and Pattern Recognition, 2005.

[14] E. Mazor, A. Averbuch, Y. Bar-Shalom, and J. Dayan, "Interactive multiple model methods in target tracking: A survey," IEEE Transactions on Aerospace and Electronic Systems, vol. 34, pp. 103-123, 1998.

[15] S. Kim, H. Oh, and A. Tsourdos, "Nonlinear model predictive coordinated standoff tracking of moving ground vehicle," AIAA Guidance, Navigation, and Control Conference, Potland, Oregon, USA, 2011.

[16] K. Mehrotra and P. R. Mahapatra, "A jerk model for tracking highly maneuvering targets," IEEE Transactions on Aerospace and Electronic Systems, vol. 33, no. 4, pp. 1094-1105, 1997.

[17] B. White, A. Tsourdos, I. Ashokaraj, S. Subchan, and R. Zbkowski, "Contaminant cloud boundary monitoring using network of UAV sensors," IEEE Sensors Journal, vol. 8, no. 10, pp. 1681-1692, 2008.

[18] M. Zhang, S. Knedik, and O. Loffeld, "An adaptive road-constrained IMM estimator for ground target tracking in GSM networks," International Conference on Information Fusion, 2007.

[19] M. Arulampalam, N. Gordon, M. Orton, and B. Ristic, "A variable structure multiple model particle filter for GMTI tracking," International Conference on Information Fusion, 2002.

[20] M. Tahk and J. Speyer, "Target tracking problems subject to kinematic constraints," IEEE Transactions on Automatic Control, vol. 35, no. 3, pp. 324-326, 1990.

[21] D. Simon and T. Chia, "Kalman filtering with state equality constraints," IEEE Transactions on Aerospace and Electronic Systems, vol. 38, no. 1, pp. 128-136, 2002.

[22] F. L. Lewis, Applied optimal control and estimation. Prentice Hall Englewood Cliffs, NJ, 1992.

[23] C. Yang, M. Bakich, and E. Blasch, "Nonlinear constrained tracking of targets on roads," International Conference on Information Fusion, 2005.

[24] C. Rasmussen and C. Williams, Gaussian Process for Machine Learning. MIT Press, 2006.

[25] S. Limited, "S-paramics software." http://www.sias.com, Jan 2011.

[26] T. Kirubarajan, Y. Bar-Shalom, K. Pattipati, and I. Kadar, "Ground target tracking with variable structure imm estimator," IEEE Transactions on Aerospace and Electronic Systems, vol. 36, no. 1, pp. 26-46, 2000. 